\title{
Molecular methods: are their results of help or do they make more confuse the clinical management of patients?
}

\author{
Anna Pierro, ${ }^{1}$ Vittorio Sambri, ${ }^{1,2}$ \\ ${ }^{1}$ Microbiology Unit, Romagna Local Health Unit, Pievesestina; ${ }^{2}$ DIMES, University of Bologna, Italy
}

\begin{abstract}
The introduction of molecular methods has revolutionised the diagnostic microbiology in the last 5 years. This new paragdigm is basically changing the laboratory diagnosis since these techniques do not identify any more antigens, but genetic sequences specific for each selected microrganism. The introduction of the polymerase chain reaction (PCR) techniques and other nucleic acid based amplification technologies (NAATs) has opened a new era by allowing detection of microorganisms that were previously difficult or impossible to detect by traditional microbiological methods. Later, with the advent of multiplex PCR, real-time PCR and then microarray technology and improvements in efficiency through automation, the role of molecular methods has further increased and this development is still going on (11).

The field of application of the molecular methods includes all the branches of microbiology with extensive implementation also in epidemiology and infection control.

The molecular biological techniques has been therefore introduced in the different diagnostic algorithms for the identification of the different etiologic agents of infection: this has lead to the possibility of identifing pathogens in the very early stages of infection. Moreover, molecular methods have also allowed to identify microorganisms whose isolation is difficult or yet not achievable by conventional methods (9). In the field of virology, PCR technology has now only an intermediate position between the viruses isolation in cell culture that has
\end{abstract}

Correspondence: Anna Pierro, Microbiology Unit, Romagna Local Health Unit, Piazza della Liberazione 60, 47522 Pievesestina (FC), Italy. Tel.: +39.0547.39.4808 - Fax: +39.0547.39.4801.

E-mail: anna.pierro@austromagna.it

Key words: Laboratory diagnosis; Molecular methods; Infection.

Contributions: AP wrote the manuscript, VS revised the paper.

Conflict of interest: the authors declare no potential conflict of interest.

Received for publication: 19 December 2016.

Accepted for publication: 19 December 2016.

(C) Copyright A. Pierro and V. Sambri, 2016

Licensee PAGEPress, Italy

Microbiologia Medica 2016; 31:6494

doi:10.4081/mm.2016.6494

This article is distributed under the terms of the Creative Commons Attribution Noncommercial License (by-nc 4.0) which permits any noncommercial use, distribution, and reproduction in any medium, provided the original author(s) and source are credited. well known drawbacks and difficulties and the serological tests that are very often unhelpful in achieving a complete diagnosis. Similarly, in the field of bacteriology, many different molecular methods have been applied to the detection of bacteria for which culture is difficult or impossible in the routine microbiology laboratory. Moreover, the sensitivity and time requirement of culture based techniques are largely influenced by many factors (e.g. previously and/or concurrent antimicrobial treatment). The use of molecular methods has been also proven to greatly enhance the sensitivity of tests for stool and blood parasites, since most of these organisms are not routinely cultivable and microscopy or serology are by far less sensitive and quite often of limited clinical relevance.

The molecular technologies have also been included in the routine workflow for the monitoring of several viral infections, such as HCV, HIV and HBV. In the filed of virology the molecular quantification of the load of viral nucleic acid is currently the corner stone used to stage disease activity, to predict the progession of the disease and to monitor the efficacy of antiviral specific therapy $(5,7,8,13)$. In bacteriology this methods can be applied to those severe bacterial infections that require an early diagnosis for the rapid implementation of a pathogen driven antibiotic therapy, such as sepsis. In addition the elevated sensitivity and rapidity and the independence from the use of antimicrobic drugs makes these methods the ideal techniques to identyify all the cases in which a chemoprophylactic intervention plays a crucial role in the prevention of secondary infections among close contacts such as for meningococcal disease $(2,3,12)$.

Moreover, molecular methods have been applied to the study of HCV and HIV genotyping that is of outmost importance for the implementation of specific therapeutic protocols. Genotyping by molecular methods is also the basic tool for the identification of the oncogenic genotypes of HPV $(1,4)$. In general, nowadays the detection of specific bacterial genes that cause phenotypic resistance to antibacterial drugs or the identification of antiviral drug resistance related SNPs in the genome of either HCV and HIV are only ideintified by nucleic acid amplification and sequence analysis.

The use of molecular technologies has been recently very useful in the management of emerging (or re-emerging) infectious disease, since their rapidity and sensitivity can be of great help in the rapid investigation and management of outbreaks. The advent of molecular epidemiology has thus revolutioned how outbreaks are investigated and managed.

The use of molecular tecniques (based on amplification and sequencing) has recently become a commonly used approach to identify bacteria and fungi from clinical samples: these methods are generally targeted at the $16 \mathrm{~S}$ sRNA bacterial gene or $18 \mathrm{~S}$ rRNA fungal gene that are considered as very conserved region of the genome, so that the amplicons are Sanger sequenced and identification is achieved by comparing the obtatained strand with those available from many different web data bases (6). In the last three years, the Next Generation 
Sequencing (NGS) technologies have been introduced step after step also in the field of clinical microbiology: these innovative techniques are capable to provide data from sequence of massive amount of DNA, when compared with the classical Sanger method (10).

In addition to the higher sensitivity and the faster turn around time compared with culture based methods, molecular techniques can identify multiples targets in one single run, as for the use of microarrays and multiplex real-time amplification. These multiparametric methods allow to identify simultaneously a range of pathogens for each individual single disease: this is what today is defined as the syndromic microbiologic approach that has demonstrated a proven efficacy in sexually transmissible and respiratory infections.

Molecular methods have, therefore, transformed the diagnostic approach to infections and the way in which laboratories identify the pathogenic agents. These methods are more sensitive, more specific, faster and can identify simultaneously more pathogens, compared to the most traditional methods: at the overall evaluation is reasonable to state that the introduction of these technologies are decreasing the hospital length of stay, optimising the treatment selection and the antimicrobial stewardship. Given the daily increasing use of molecular techniques in the microbiologic workflow, the cost of molecular methods is decreasing day after day thus allowing a widespread use. The introduction of point of care (POC) tests, for example, has allowed the use of molecular methods also in developing countries where the laboratory resources are limited. Apparently the wide introduction of molecular techniques have brought several advantages in the field of diagnostic microbiology, with a few drawbacks. The most relevant of these disadvanteges is nowadays strongly emerging in the daily clinical practice: how should we to interpret the results obtained by some of these molecular methods? What is the relevant information provided?

It is now becoming more and more common that more than one potentially pathogenic microrganism is detected by real time multiplex PCR methods applied to syndromic infection. Thus identication of more a than one genomic specific sequence belonging to pathogenic or even only potentially pathogenic germs has opened a wide debate about the clinical meaning of these results: should we re-think the etiologic concepts about the causes of infective illness such as the gastro intestinal syndrome or the upper respiratory tract infections? Do we have any clue to establish if these multiple identifications are truly related to the etiology of the infections or they are rather generated by something similar to just colonization? Another intriguing hypotesis to explain these potential multiple etiologies for diseases that have been until the advent of molecular methods just linked to one single microrganism could be the persistance of genomic fragment after the disruption of the microbes: no data are available about how long this phenomenon of genetic persistance could last in selected districts of the human bodies.

Another important issue related to the management of data derived from the implementation of high throughput technologies, such as the NGS, to the microbiologic workflows is related to the best way to manage such an enormous amount of information: of course the best option is currently to set up a collaborative plan with bioinformatic experts. Of course this approach could only be fruitful if the microbiologists would work in a strict and reciprocal way with bioinformatics.

The wide use of molecular techniques even in bacteriology has prompted to the necessity to coin a new definition for the detection of bacterial genome segments into clinical samples, and in particular in primary blood specimens: DNAemia. Now, what could be the true clinical meaning of DNAemia? It is of extreme relevance to underline that this result is likely of no clinical significance in the absence of signs and symptoms of bacteriemia and invasive infection. Evidence has been provided that the detection of bacterial DNA sequences is a quite common feature even in healthy blood donors.
This consideration, overall, open a question: how can infection be differentiated from disease since the presence of nucleic acid does not necessarily correlate with the presence of viable organism? Additionally, the identified microorganisms could be commensal, colonizing bacteria or viruses that are not contributing to the clinical disease. Consequently it is becoming more and more crucial for the clinicians to achieve the capability to distinguish colonizers and contaminants from true pathogens, as well as to interpret the results of molecular methods. This can only be possible when a close collaboration between laboratory and clinicians is in place and effective. All the information about patients, therapy, specimens collection, storage and transport are becoming therefore crucial for the interpretation of the results obtained by using molecular methods. In other words, it is crucial to acquire the capability to interpret apparently discordant findings: e.g. blood colture results negative and molecular methods results positive for one or more microorganism, or viceversa. Nowadays, the possibility to add new test in the diagnostic workflow is likely on a monthly base and this would mean to have a larger, faster and stronger capacity to diagnose many infections. But adding new tests is also meaning to have more and more results to evaluate. Only a close collaboration among the different professionals involved can lead to a correct interpretation of the laboratory results and an optimal patient management.

\section{References}

1. Brestovac B, Harnett GB, Smith DW, et al. Multiplex nested PCR (MNP) assay for the detection of 15 high risk genotypes of human papillomavirus. J Clin Virol 2005;33:116-22.

2. Carrol ED, Thomson AP, Riordan FA, et al. Increasing microbiological confirmation and changing epidemiology of meningococcal disease on Merseyside, England. Clin Microbiol Infect 2000;6:259-62.

3. Clarke SC, Diggle MA, Edwards GF. Semiautomation of multilocus sequence typing for the characterization of clinical isolates of Neisseria meningitidis. J Clin Microbiol 2001;39:3066-71.

4. Cuzick J. Role of HPV testing in clinical practice. Virus Res 2002; 89:263-9.

5. Dax EM. The window period and HIV tests. ASHM J Club 2004;13:9-11.

6. Fredricks DN, Relman DA. Application of polymerase chain reaction to the diagnosis of infectious diseases. Clin Infect Dis 1999;29:475-86.

7. Fried MW, Shiffman ML, Reddy KR, et al. Peginterferon alpha-2a plus ribavirin for chronic hepatitis $\mathrm{C}$ virus infection. N Engl J Med 2002;347:975-82.

8 Katzenstein TL. Molecular biological assessment methods and understanding the course of the HIV infection. APMIS 2003:1-37.

9. Nolte FS, Caliendo AM. Molecular detection and identification of microorganism. In: Murray P, Baron EJ, Jorgensen JH, et al, eds. Manual of clinical microbiology. $8^{\text {th }}$ ed. Washington: ASM Press, 2003. pp 234-56.

10. Rehm HL, Bale SJ, Bayrak-Toydemir P, et al. ACMG clinical laboratory standards for next-generation sequencing. Genet Med 2013;15:733-47

11. Speers DJ. Clinical applications of molecular biology for infectious diseases. Clin Biochem 2006;27:39-51.

12. Taha, MK. Simultaneous approach for nonculture PCR-based identification and serogroup prediction of Neisseria meningitidis. J Clin Microbiol 2000;38:855-7.

13. Thomson EC, Main J. Advances in hepatitis B and C. Curr Opin Infect Dis 2004;17:449-59. 